\title{
Minimal Excitation States of Electrons in One-Dimensional Wires
}

\author{
J. Keeling, ${ }^{1}$ I. Klich, ${ }^{2}$ and L. S. Levitov ${ }^{1}$ \\ ${ }^{1}$ Department of Physics, Massachusetts Institute of Technology, 77 Massachusetts Avenue, Cambridge, Massachusetts 02139, USA \\ ${ }^{2}$ Department of Physics, California Institute of Technology, Pasadena, California 91125, USA
}

(Received 1 April 2006; published 14 September 2006)

\begin{abstract}
A strategy is proposed to excite particles from a Fermi sea in a noise-free fashion by electromagnetic pulses with realistic parameters. We show that by using quantized pulses of simple form one can suppress the particle-hole pairs which are created by a generic excitation. The resulting many-body states are characterized by one or several particles excited above the Fermi surface accompanied by no disturbance below it. These excitations carry charge which is integer for noninteracting electron gas and fractional for Luttinger liquid. The operator algebra describing these excitations is derived, and a method of their detection which relies on noise measurement is proposed.
\end{abstract}

DOI: 10.1103/PhysRevLett.97.116403

Controlling single electrons is one of the main avenues of research in nanoelectronics. Once advanced far enough, it will bring about a range of quantum-coherent singleparticle sources with full control over the orbital and spin degrees of freedom. Currently efforts are mostly focused on employing localized electron states, trapped on metal islands [1] or quantum dots [2] and shuttled between the dots or islands by electric pulses [1,2] or acoustic fields [3]. It is of interest, however, to extend the concept of singleparticle sources to the situation when electrons propagate freely as part of a degenerate Fermi system. If proved feasible, it would allow one to harness particle dynamics, characterized by high Fermi velocity, $v_{F} \sim 10^{8} \mathrm{~cm} / \mathrm{s}$, to transmit quantum states in a solid and, at low temperature, to use Fermi-Dirac statistics for generating many-particle entangled states [4].

In this Letter we propose a scheme which allows the creation of "clean" electric current pulses, free of particlehole excitations. We consider a $1 d$ electron gas, serving as a prototype for carbon nanotube, quantum wire, and point contact systems, in which current is driven by voltage pulses with a typical frequency small compared to the Fermi energy. In this quasistationary regime the electric response is described as $I(t)=g_{0} V(t)$ with $g_{0}=e^{2} / h$ the Landauer conductance. A current pulse, which carries total charge $\Delta q=g_{0} \int V(t) d t$, is a collective many-body state involving a number of fermions excited to a higher energy [5]. Microscopically, such a current pulse is described by a number of particle-hole excitations, with energies of the order $\hbar / \tau$, where $\tau$ is the duration of the pulse. As discussed in Refs. [5-7] and below, these excitations can be probed by noise measurement [8].

Here we show that, quite strikingly, by engineering the pulse profile one can inhibit the particle-hole excitations. We analyze the particle-hole content of current pulses in a single-channel conductor, and pose and solve the problem of minimizing the number of such excitations. The condition required for the excitation number to be small is area quantization, $\int V d t=n h / e$, where $n$ is an integer. We show that such pulses, carrying integer charge $q=n e$,
PACS numbers: 71.10.Pm, 03.65.Ud, 03.67.Hk, 73.50.Td

are accompanied by fewer excitations than noninteger pulses. Also, we show how to optimize the $V(t)$ profile, by designing pulses which are totally free of particle-hole excitations. Such pulses excite $n$ electrons above the Fermi level, with other electrons conspiring to fill the void and produce a complete Fermi sea, with no holes.

The properties of such "ideal" pulses may be inferred by asking for $V(t)$ that creates the minimum number of right- or left-moving excitations $N_{\mathrm{ex}}^{\alpha}=N_{e}^{\alpha}+N_{h}^{\alpha}$,

$$
N_{e}^{\alpha}=\sum_{\epsilon>\epsilon_{F}}\left\langle a_{\epsilon}^{\dagger} a_{\epsilon}\right\rangle, \quad N_{h}^{\alpha}=\sum_{\epsilon<\epsilon_{F}}\left\langle a_{\epsilon} a_{\epsilon}^{\dagger}\right\rangle,
$$

where $\alpha=r, l$ for right and left movers, and $N_{e}^{\alpha}, N_{h}^{\alpha}$ are the numbers of excited electrons and holes (Fig. 1). The sum is restricted to right- or left-moving particles, $k \approx$ $k_{F},-k_{F}$. The operators $a_{\epsilon}^{\dagger}$ create particles in the singleparticle energy eigenstates. Since $\Delta q=e\left(N_{e}-N_{h}\right)$, one may naïvely expect that $N_{\mathrm{ex}}$ is minimized for a given $\Delta q$ when $N_{h}$ or $N_{e}$ vanish.

This naïve expectation is correct, and it is possible to find a time-dependent field that excites exactly $n$ electrons above the Fermi level leaving no other disturbance in the system. The excitation number $N_{\mathrm{ex}}$, introduced in Eq. (1), can be linked to noise (see below), thus $N_{\text {ex }}$ can be mea-

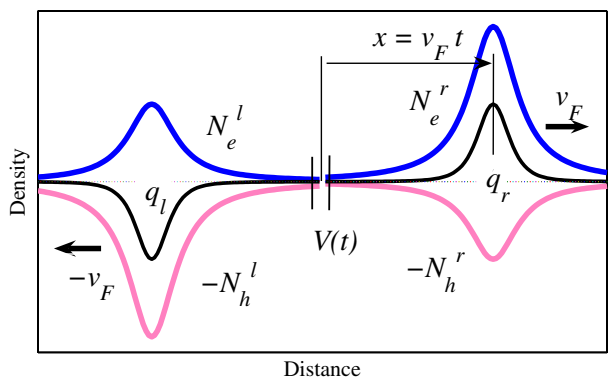

FIG. 1 (color online). Real space picture of counterpropagating electron and hole pulses, for a general time-dependent field. In the special case of Eq. (6), $N_{h}^{r}=N_{e}^{l}=0, q_{r}=N_{e}^{r}, q_{l}=$ $-N_{h}^{l}$. 
sured by sending the excited pulse on a beam splitter (point contact) and detecting scattering noise.

The many-body states for these pulses are found below to have a simple direct product form. For the right-moving electrons, these states are

$$
|\psi\rangle=\prod_{m=1}^{n} A_{m}^{\dagger}|0\rangle, \quad A_{m}^{\dagger}=\sqrt{2 \tau_{m}} \sum_{\epsilon>\epsilon_{F}} e^{-\xi_{m} \epsilon / \hbar} a_{\epsilon}^{\dagger},
$$

where $|0\rangle$ is the undisturbed Fermi sea, and

$$
\xi_{m}=\tau_{m}-i t_{m}, \quad \tau_{m}>0, \quad m=1, \ldots, n,
$$

are complex parameters specifying each pulse width and the creation moment. Thus, a single operator $A_{m}^{\dagger}$ creates an electron in a superposition of single-particle eigenstates with $k \approx k_{F}$. The form in Eq. (2) applies both to a dispersionless system, $\epsilon=v_{F} k$, and to the more general case of a one to one relation between $|k|$ and $\epsilon$.

The product form and the absence of holes at $\epsilon<\epsilon_{F}$ in (2) means that the particles excited by different operators $A_{m}^{\dagger}$ are not entangled with one another, and that the excitation leaves the Fermi sea intact. Each of the particles (2) has an exponential energy distribution, $p(\epsilon) \propto e^{-\beta_{m} \epsilon}$, of the width determined by inverse pulse duration: $\beta_{m}=$ $2 \tau_{m} / \hbar$. From our analysis it follows that this is the only kind of energy distribution possible under the requirement that the Fermi sea remains undisturbed.

The remarkable and somewhat paradoxical property of the states (2) is "charge imbalance," i.e., $n$ particles above Fermi level with, apparently, no accompanying holes. It will be seen below that such states can nevertheless be created by electromagnetic pulses in a realistic experimental situation. The accompanying holes in fact do appear near the Fermi level, however, at the point $-k_{F}$ opposite to where electrons are created. A large momentum transfer $2 n \hbar k_{F}$ associated with an excitation which is slow on the scale of $\epsilon_{F}$ can be understood as a fermion anomaly, a result of the collective response of the entire Fermi sea.

It is instructive to consider the real space profile of these states. Using $\psi(x, t)=\psi\left(x-v_{F} t\right)$, for the case of a dispersionless Fermi system, the real space profile of the single-particle state that the operator $A_{m}^{\dagger}$ creates is given by the Fourier transform of the energy representation, Eq. (2). For $n=1$ this gives

$$
\psi(x, t)=\sqrt{\frac{v_{F}}{2 \pi}} \frac{i \sqrt{2 \tau_{1}}}{x-v_{F}\left(t-t_{1}\right)+i v_{F} \tau_{1}},
$$

i.e., the final many-body state contains one extra electron in a state with a Lorentzian density profile, matching the time dependence of the voltage pulse, as discussed below. Similarly, there is a counterpropagating opposite sign (hole) pulse, as illustrated in Fig. 1.

If the time-dependent voltage is applied across a short interval (Fig. 1), such that the time of transit through the ac field region is short compared to the pulse width $\tau$, then one may consider an instantaneous scattering approximation, which for a dispersionless Fermi system and right-moving particles with $k \approx k_{F}$, can be written in terms of the singleparticle Hamiltonian,

$$
H=-i \hbar v_{F} \partial_{x}+\delta(x) \hbar v_{F} \phi(t), \quad \frac{d \phi}{d t}=-\frac{e}{\hbar} V(t) .
$$

Thus, the single-particle states on either side of $x=0$ are related by time-dependent forward scattering phase, $\psi\left(x^{+}, t\right)=\psi\left(x^{-}, t\right) e^{i \phi\left(t-x / v_{F}\right)}$. The forward scattering phase $e^{i \phi(t)}$, describing the effect of $V(t)$ on single-particle wave functions, defines a canonical transformation $U$ of fermion operators in the many-body problem:

$$
a_{\epsilon}=\sum_{\epsilon^{\prime}}\left\langle\epsilon|U| \epsilon^{\prime}\right\rangle \tilde{a}_{\epsilon^{\prime}}, \quad\left\langle\epsilon|U| \epsilon^{\prime}\right\rangle=\int e^{i\left(\epsilon-\epsilon^{\prime}\right) t / \hbar} e^{i \phi(t)} d t .
$$

Substituting in Eq. (1) and summing over the Fermi vacuum, we find the excited electron and hole numbers

$$
N_{e}=\sum_{\epsilon>\epsilon_{F}>\epsilon^{\prime},}\left|\left\langle\epsilon|U| \epsilon^{\prime}\right\rangle\right|^{2}, \quad N_{h}=\sum_{\epsilon<\epsilon_{F}<\epsilon^{\prime},}\left|\left\langle\epsilon|U| \epsilon^{\prime}\right\rangle\right|^{2} .
$$

This means that the clean pulse condition, $N_{h}=0$, is expressed mathematically as Fourier harmonics $\int e^{i \omega t} e^{i \phi(t)} d t$ vanishing at $\omega<0$, where $\hbar \omega=\epsilon-\epsilon^{\prime}$.

Let us now show that clean states can be obtained by applying a sum of Lorentzian pulses of quantized area:

$$
e^{i \phi(t)}=\prod_{m=1}^{n} \frac{t+i \xi_{m}^{*}}{t-i \xi_{m}}, \quad V(t)=\frac{\hbar}{e} \sum_{m=1}^{n} \frac{-2 \tau_{m}}{\left(t-t_{m}\right)^{2}+\tau_{m}^{2}} .
$$

By Fourier transforming $e^{i \phi(t)}$ which is analytic in the lower half plane of complex $t$ we see that the negative Fourier harmonics vanish. For example, at $n=1$,

$$
\left\langle\epsilon|U| \epsilon^{\prime}\right\rangle=\int \frac{d t}{2 \pi} \frac{t+i \xi_{1}^{*}}{t-i \xi_{1}} e^{i \omega t}=\delta(\omega)-2 \tau_{1} e^{-\omega \xi_{1}} \theta(\omega) .
$$

Thus we have $N_{h}=0$ and $N_{e}=n$, proving that the time dependence (6) indeed leads to clean pulses. Sign reversal in (6), $\phi, V \rightarrow-\phi,-V$, gives pulses which create $n$ holes in a similar clean fashion.

The form of Eq. (6) is suggested by recalling several previous instances when Lorentzian $V(t)$ of quantized area appeared, such as tunneling of phase in Josephson junctions [9], charge pumping noise [10], quenching of Coulomb blockade [11], 1d quantum hydrodynamics [12], and vertex operators in quantum Hall systems [13]. Besides producing particularly low scattering noise [10], the pulses (6) were found to give rise to strikingly simple counting statistics $[14,15]$. The reason for the latter, which previously was unknown, will be clarified below.

The clean states obtained by applying an electric field with the time dependence (6) are of the form described in Eq. (2). We first show it in the case $n=1$ (total phase increase $2 \pi$ ), for simplicity setting $\hbar=1$. The initial state, i.e., the filled Fermi sea, is associated with a projection $\hat{n}$ on the states with $\epsilon<\epsilon_{F}$ in the single-particle Hilbert space. After applying a unitary evolution $U$, the evolved 
Fermi sea will be associated with $\hat{n}^{\prime}=U \hat{n} U^{\dagger}$. The particles taken from below to above the Fermi surface are thus associated with $U_{+-}=(1-\hat{n}) U \hat{n}$, which in the current case, described by Eq. (7), has the form:

$$
\left\langle\epsilon\left|U_{+-}\right| \epsilon^{\prime}\right\rangle=-2 \tau_{1} e^{-\left(\epsilon-\epsilon^{\prime}\right) \xi_{1}}=-2 \tau_{1} e^{-\epsilon \xi_{1}} e^{\epsilon^{\prime} \xi_{1}} .
$$

Owing to multiplicativity of exponential, $U_{+-}$is a rank one matrix, and as will be shown next, this means that only a single particle is excited, ensuring the minimal excitation property.

The requirement that the matrix is of rank one, which is basis independent, requires $U_{+-}$to be of the form $U_{+-}=$ $c\left|\phi_{+}\right\rangle\left\langle\phi_{-}\right|$, with the states $\phi_{-}, \phi_{+}$inside and outside the Fermi sea. This structure implies there can be at most one particle excited above the Fermi surface. Consider a hypothetical transition in which two or more particles are excited from levels below to above the Fermi surface. For any given pair of initial levels below the Fermi surface, $a, b$, and final levels above the Fermi surface, $a^{\prime}, b^{\prime}$, there are two ways such transition can be achieved; $a \rightarrow a^{\prime}, b \rightarrow$ $b^{\prime}$ and $a \rightarrow b^{\prime}, \quad b \rightarrow a^{\prime}$. However, since $U_{m \rightarrow m^{\prime}}=$ $\left\langle m^{\prime}\left|U_{+-}\right| m\right\rangle=c\left\langle m^{\prime} \mid \phi_{+}\right\rangle\left\langle\phi_{-} \mid m\right\rangle$ for any pair of states $|m\rangle,\left|m^{\prime}\right\rangle$ it follows that the two-fermion transition amplitude vanishes:

$$
U_{a \rightarrow a^{\prime}} U_{b \rightarrow b^{\prime}}-U_{a \rightarrow b^{\prime}} U_{b \rightarrow a^{\prime}}=0 .
$$

Thus the Fermi statistics blocks two-particle transitions. Similarly, the requirement for having no more than $n$ particles or holes excited is that $U_{+-}$is a matrix of rank $n$.

The $\delta(\omega)$ term in Eq. (7) might suggest there is a finite probability that the pulses (6) produce no excitation. However, the corresponding weight is exponentially small in the pulse width, $\tau \epsilon_{F} / \hbar \gg 1$, and thus can be ignored. Thus, a single particle is created in the state $\left|\phi_{+}\right\rangle$, and by matching the above form of $U_{+-}$one finds that the state created $\left|\phi_{+}\right\rangle$is that of Eq. (2). Since $U_{-+}=0$, it is clear there are no holes created moving to the right. In contrast, the left-moving particles have $U_{+-}=0$ and $U_{-+}$of rank one. Thus there is one hole created near $-k_{F}$ in momentum space, but no particles excited.

Having understood the single pulse, one can now consider combining such pulses. Consider two pulses,

$$
e^{i \phi(t)}=\left(\frac{t+i \xi_{1}^{*}}{t-i \xi_{1}}\right)\left(\frac{t+i \xi_{2}^{*}}{t-i \xi_{2}}\right) .
$$

Using the result of a single pulse acting on the vacuum state, and introducing $\tilde{A}_{2}^{\dagger}=U_{1} A_{2}^{\dagger} U_{1}^{\dagger}$ [i.e., transforming each single-particle operator in $A_{2}^{\dagger}$ by the unitary matrix $U_{1}$ as in Eq. (5)], we can write the result of two pulses as: $U_{1} U_{2}|0\rangle=U_{1} A_{2}^{\dagger}|0\rangle=U_{1} A_{2}^{\dagger} U_{1}^{\dagger} U_{1}|0\rangle=\tilde{A}_{2}^{\dagger} A_{1}^{\dagger}|0\rangle$. Using the matrix elements, Eq. (7), we find

$$
\begin{aligned}
\tilde{A}_{2}^{\dagger} & =A_{2}^{\dagger}-2 \tau_{1} \sqrt{2 \tau_{2}} \int_{0}^{\infty} d \nu \int_{0}^{\infty} d \omega e^{-\omega \xi_{1}-\nu \xi_{2}} a^{\dagger}(\omega+\nu) \\
& =A_{2}^{\dagger}-\frac{2 \tau_{1}}{\xi_{1}-\xi_{2}}\left(A_{2}^{\dagger}-\sqrt{\tau_{2} / \tau_{1}} A_{1}^{\dagger}\right)
\end{aligned}
$$

Since the operators $A^{\dagger}$ are fermionic, $A_{1}^{\dagger 2}=0$, and so the result of two phase pulses is given by:

$$
U_{1} U_{2}|0\rangle=\frac{\xi_{1}^{*}+\xi_{2}}{\xi_{2}-\xi_{1}} A_{2}^{\dagger} A_{1}^{\dagger}|0\rangle
$$

Similarly, for $n$ pulses, Eq. (6), we obtain the algebra

$$
\prod_{m=1}^{n} U_{m}|0\rangle=\prod_{m>m^{\prime}} \frac{\xi_{m^{\prime}}^{*}+\xi_{m}}{\xi_{m^{\prime}}-\xi_{m}} A_{n}^{\dagger} A_{n-1}^{\dagger} \cdots A_{1}^{\dagger}|0\rangle .
$$

The expression for holes near $-k_{F}$ is similar, but with the complex conjugate of the factor multiplying the operators $A_{m}^{\dagger}$. When the creation times are equal $t_{m}=t_{m}^{\prime}$, the prefactor in Eq. (12) simplifies: it becomes real, depends only on $\tau_{m}$, and is antisymmetric in permutations. This means that even looking at just the Fermi point $k_{F}$, the pulse creation operators effectively commute (since $A_{m}^{\dagger}$ anticommute). Remarkably, the form of the expression (12) is similar to the Laughlin state in a quantum Hall system.

In the limit $\xi_{m} \rightarrow \xi_{m^{\prime}}$ the prefactor in Eq. (12) appears to diverge; however, the product of two identical single fermion operators would vanish. Taking these limits together, the result is expressed through $A_{m}^{\dagger} \partial_{\xi_{m}} A_{m}^{\dagger}$, i.e., is nonzero, but not of the form of Eq. (2).

Similarly, a pulse and an antipulse give:

$$
e^{i \phi(t)}=e^{-i \phi_{1}(t)} e^{i \phi_{2}(t)}=\left(\frac{t-i \xi_{1}}{t+i \xi_{1}^{*}}\right)\left(\frac{t+i \xi_{2}^{*}}{t-i \xi_{2}}\right),
$$

with $\tau_{1}, \tau_{2}>0$. This combination corresponds to zero total phase change, and thus the associated pulse carries zero net current. Such a pulse exhibits a higher degree of particlehole entanglement than that of Eq. (2).

Introducing the operator $B_{1}$, creating a single hole below the Fermi level near $k_{F}$, to describe $\bar{U}_{1}$ corresponding to $e^{-i \phi_{1}(t)}$, one can follow the same procedure as in Eq. (10):

$$
\begin{aligned}
\bar{U}_{1} U_{2}|0\rangle & =\left(\bar{U}_{1} A_{2}^{\dagger} \bar{U}_{1}^{\dagger}\right) B_{1}|0\rangle \\
& =\frac{\xi_{2}-\xi_{1}}{\xi_{2}+\xi_{1}^{*}} A_{2}^{\dagger} B_{1}|0\rangle-\frac{2 \sqrt{\tau_{1} \tau_{2}}}{\xi_{2}+\xi_{1}^{*}}|0\rangle,
\end{aligned}
$$

where as in Eq. (10), $\bar{U}_{1} A_{2}^{\dagger} \bar{U}_{1}^{\dagger}$ has the form of $u A_{2}^{\dagger}+v B_{1}^{\dagger}$, with the last term, applied to the state $B_{1}|0\rangle$, producing $|0\rangle$. Hence the final state is a superposition containing two parts: the unperturbed Fermi sea, and an electron-hole pair $A_{2}^{\dagger} B_{1}$ with an extra electron just above the Fermi level and a hole just below. As $\tau_{1}, t_{1} \rightarrow \tau_{2}, t_{2}$ the weight of the excited part decreases, and the state approaches the unperturbed state.

It is interesting to generalize the above results to Luttinger liquids, where charge fractionalization [16] allows one to create clean pulses carrying noninteger charge. Here we consider chiral Luttinger liquid of the kind realized on a quantum Hall edge. In the simplest case of a single chiral mode the Largrangian [17] for displacement field coupled to an ac voltage is of the form 


$$
\mathcal{L}=\int\left[\frac{1}{4 \pi} \partial_{x} \theta\left(\partial_{t}-v \partial_{x}\right) \theta+\frac{\sqrt{\nu}}{2 \pi} \partial_{x} \theta e V(t, x)\right] d x .
$$

The operators $\psi(t, x) \propto e^{i \sqrt{\nu} \theta(t, x)}(\nu=1 / m$ for Laughlin state) describe quasiparticles of charge $e_{*}=\nu e$ which obey Fermi statistics [17]. Solving for the displacement operator time dependence, $\left(\partial_{t}-v \partial_{x}\right) \theta=-\sqrt{\nu} e V(t, x)$, we obtain the Heisenberg evolution of a quasiparticle:

$$
\psi(t, x)=\psi(0, x+v t) \exp \left[-i \int_{0}^{t} e_{*} V\left(t^{\prime}, x^{\prime}\right) d t^{\prime}\right],
$$

$x^{\prime}=x+v\left(t-t^{\prime}\right)$. This means that the phase picked up after passing through the ac field is $\delta \phi=-\int e_{*} V\left(t^{\prime}\right) d t^{\prime}$ (at fast passage, i.e., when $\tau v \gg L$, where $L$ is the width of the region where field is applied). From Eq. (16) it is clear that the problem maps exactly onto the free fermion problem studied above, with electrons replaced by fractional charge quasiparticles. The optimal pulses (phase increase $2 \pi$ ) are Lorentzians of area $\int V d t=h / \nu e$, with the corresponding excitation carrying charge $e_{*}$.

To discuss the possibility of testing the above predictions experimentally, let us link the excitation number with noise. The operator counting particle-hole excitations, $\hat{N}_{\mathrm{ex}}=\sum_{\epsilon<\epsilon_{F}} a_{\epsilon} a_{\epsilon}^{\dagger}+\sum_{\epsilon>\epsilon_{F}} a_{\epsilon}^{\dagger} a_{\epsilon}$, has an expectation value which can be found from the trace of $\hat{N}_{\text {ex }}$ with the singleparticle density matrix describing the system after the field is applied. Writing this trace in the time domain, so the applied field is described by a time-dependent phase, one has:

$$
\begin{aligned}
\left\langle\hat{N}_{\mathrm{ex}}\right\rangle & =\int d t \int d t^{\prime} N_{\mathrm{ex}}\left(t, t^{\prime}\right)\left[e^{-i \phi\left(t^{\prime}\right)} \rho_{0}\left(t^{\prime}, t\right) e^{i \phi(t)}\right] \\
& =\sum_{ \pm} \int \frac{d t}{2 \pi} \int \frac{d t^{\prime}}{2 \pi} \frac{\pi^{2} T^{2} e^{i\left[\phi(t)-\phi\left(t^{\prime}\right)\right]}}{\sinh ^{2} \pi T\left(t-t^{\prime}-i 0^{ \pm}\right)} \\
& =\int \frac{d \omega}{4 \pi^{2}}\left|\int e^{i \phi(t)+i \omega t} d t\right|^{2} \omega \operatorname{coth} \frac{\omega}{2 T},
\end{aligned}
$$

where the average is taken over a state with temperature $T$. This expression is equal, up to a system-dependent factor, to the noise created as a result of the pulse scattering on a barrier [10]. Thus one can measure $N_{\mathrm{ex}}$ directly by passing the excited pulse through a beam splitter, such as point contact, and detecting resulting current noise.

For nonquantized pulses (phase increase not a multiple of $2 \pi$ ), one has $\int e^{i \phi(t)+i \omega t} d t \sim 1 / \omega$, and so $N_{\mathrm{ex}}$ exhibits a $\log$ divergence [10]. The quantized pulses, characterized by smaller $N_{\text {ex }}$, produce lower noise. Among those the Lorentzian pulses (6) provide absolute minimum to the noise.

The noise-free character of the states (2), comprised of a few particles and undisturbed Fermi sea, makes the effect of their scattering very simple to interpret. If no dc voltage is present, i.e., the Fermi level is the same in all reservoirs, as in the above discussion, scattering of the states (2) will leave the filled Fermi sea intact, and the only contribution will arise from the excited particles. The noise in such a case, as well as the entire counting statistics, should be of single-particle character. This property of Lorentzian voltage pulses applied to the barrier was noted in Refs. [10,14].

To summarize, we described a realization of a source of single fermions which does not rely on electron confinement. The particles can be excited directly out of a Fermi sea by a carefully designed perturbation without creating additional noise. The profile of pulses required for this is Lorentzian, with quantized area and duration shorter than $\hbar / k T$ and long compared to $\hbar / \epsilon_{F}$. For typical values $T=$ $10 \mathrm{mK}$ and $\epsilon_{F}=10 \mathrm{meV}$ this gives a realistic frequency range $200 \mathrm{MHz}<\nu<2 \mathrm{THz}$. In a Luttinger liquid this provides a source of quasiparticles with fractional charge. The excited particles propagate ballistically and can be used to transmit quantum states across the system, create entangled particle-hole pairs, and can be analyzed by noise measurement.

This work was supported by NSF-NIRT DMR-0304019; J. K. acknowledges financial support from the Lindemann Trust.

[1] P. Delsing et al., Phys. Rev. Lett. 63, 1861 (1989); L. J. Geerligs et al., Phys. Rev. Lett. 64, 2691 (1990).

[2] L. P. Kouwenhoven et al., Phys. Rev. Lett. 67, 1626 (1991).

[3] J. M. Shilton et al., J. Phys. Condens. Matter 8, L531 (1996).

[4] C. W. J. Beenakker, cond-mat/0508488; P. Samuelsson, E. V. Sukhorukov, and M. Büttiker, Phys. Rev. Lett. 91, 157002 (2003); C. W. J. Beenakker et al., Phys. Rev. Lett. 91, 147901 (2003); C. W. J. Beenakker, M. Titov, and B. Trauzettel, Phys. Rev. Lett. 94, 186804 (2005); A. V. Lebedev, G. B. Lesovik, and G. Blatter, Phys. Rev. B 72, 245314 (2005).

[5] V.S. Rychkov, M. L. Polianski, and M. Büttiker, Phys. Rev. B 72, 155326 (2005).

[6] M. L. Polianski, M. G. Vavilov, and P. W. Brouwer, Phys. Rev. B 65, 245314 (2002).

[7] G. B. Lesovik and L. S. Levitov, Phys. Rev. Lett. 72, 538 (1994).

[8] R. J. Schoelkopf et al., Phys. Rev. Lett. 80, 2437 (1998); L.-H. Reydellet et al., Phys. Rev. Lett. 90, 176803 (2003).

[9] S.E. Korshunov, Pis'ma Zh. Eksp. Teor. Fiz. 45, 342 (1987) [JETP Lett. 45, 434 (1987)].

[10] H.-W. Lee and L.S. Levitov, cond-mat/9507011; condmat/9312013.

[11] Yu. V. Nazarov, Phys. Rev. Lett. 82, 1245 (1999).

[12] E. Bettelheim, A. G. Abanov, and P. Wiegmann, cond-mat/ 0606778.

[13] M. Stone, Int. J. Mod. Phys. B 5, 509 (1991).

[14] D. A. Ivanov, H.-W. Lee, and L. S. Levitov, Phys. Rev. B 56, 6839 (1997).

[15] N. d'Ambrumenil and B. Muzykantskii, Phys. Rev. B 71, 045326 (2005).

[16] C. L. Kane and M. P. A. Fisher, Phys. Rev. Lett. 72, 724 (1994); 68, 1220 (1992).

[17] X. G. Wen, Phys. Rev. B 41, 12838 (1990). 